International Journal of Translational

Medical Research and Public Health (2020), Volume 4, Issue I, 56-63

\begin{tabular}{|c|c|c|}
\hline & & $\begin{array}{l}\text { INTERNATIONAL JOURNAL OF TRANSLATIONAL } \\
\text { MEDICAL RESEARCH AND PUBLIC HEALTH } \\
\text { ISSN 2576-9499 (Online) }\end{array}$ \\
\hline l]TMRPH & Available online at www.ijtmrph.org & $\begin{array}{l}\text { ISSN 2576-9502 (Print) } \\
\text { DOI: I0.21 106/ijtmrph.133 }\end{array}$ \\
\hline
\end{tabular}

\title{
ORIGINALARTICLE | LUMBAR PUNCTURE Lumbar Puncture in Saudi Arabia: Knowledge, Awareness, Acceptance and its Refusal Among Health Professionals
}

\author{
Amani A. Alrehaili, $\mathbf{P h D}^{1 \otimes}$ \\ 'Clinical Laboratory Sciences Department, College of Applied Medical Sciences, Taif University, Al Mutamarat Rd, Al Mathnah, At Taif 2652I, Saudi Arabia \\ ${ }^{\square}$ Corresponding author email:Amani.reh@gmail.com
}

\begin{abstract}
BACKGROUND AND OBJECTIVES: Lumbar puncture (LP) is a standard technique to acquire cerebrospinal fluid (CSF) for either diagnostic or therapeutic management of neurological disorders. This study assesses the awareness and level of acceptance of LP among medical laboratory students. The study also investigates the level of understanding of the purpose of performing LP and determines the acceptance rate among the study population.

METHODS: A cross-sectional study was conducted in Taif, Saudi Arabia, using an online questionnaire distributed among medical laboratory students attending the College of Applied Medical Sciences at Taif University. Of over 200 questionnaires distributed, 176 students responded. The questionnaire queried students' sociodemographic information, their knowledge, acceptance rate, and possible complications and precautions concerning LP.A univariate analysis was performed.

RESULTS: The study showed that $81.8 \%$ of participants were aware of LP.About $53.6 \%$ of participants received their information from their education. The participants' understanding of the side effects and precautions surrounding LP operations was significantly high at $87.5 \%$ and $72.7 \%$, respectively. In all, $65.9 \%$ of participants reported their acceptance compared to $34.1 \%$ of participants who did not accept LP. In contrast, about $\mathbf{7 2 . 7 \%}$ of participants preferred not to do LP, even if the LP was prescribed by their doctor.

CONCLUSION AND IMPLICATIONS FOR TRANSLATION: The study showed high knowledge of the purpose, process, and duration of the LP procedure among clinical laboratory students. The key reasons for the rejection of the LP operation, for the majority of participants, were fear of injection and side effects, notably paralysis. This research demonstrated the need to raise proficiency in implementation and awareness of LP using methods such as training courses, academic seminars, and social media.
\end{abstract}

Key words: Lumbar puncture; Cerebrospinal fluid; Taif; Saudi Arabia; Medical laboratory students.

Copyright (C) 2020 Alrehaili. Published by Global Health and Education Projects, Inc. This is an open-access article distributed under the terms of the Creative Commons Attribution License CC BY 4.0. 


\section{Introduction}

Lumbar puncture (LP), also known as the spinal tap, is a standard technique for either diagnostic or therapeutic of neurological disorders to acquire cerebrospinal fluid (CSF).' The choroid plexus secretes CSF continuously within the brain's ventricles and the CSF circulates through channels in the brain's subarachnoid area. CSF is produced at $0.2-0.7 \mathrm{~mL} / \mathrm{min}(500-700 \mathrm{~mL} /$ day $)$ and for the adult, the average CSF volume is about $140 \mathrm{~mL}$.' Primary care doctors also perform LP since CSF is a valuable central nervous system (CNS) diagnostic tool, for diseases associated with CNS, anesthesiology, oncology, and geriatrics. ${ }^{2}$ Typical CSF tests involve protein and glucose levels, differential and cell counts, microscopic inspections, and cultivation. More testing may also be carried out such as opening strain, supernatant color, latex agglutination, and the polymerase chain reaction. ${ }^{3}$ Understanding which tests to order and how to interpret these tests allows doctors to use CSF as a main diagnostic method and for different various diseases. ${ }^{3}$ The CSF approach involves inserting a needle securely below the spinal cord into the subarachnoid area of the lumbar sac. ${ }^{4}$ Headaches, back pains, pneumonia, lower limb fatigue, subdural hematoma, bleeding and cerebrospinal fluid leakage from the site of puncture, nerve damage, or brain herniation may be complications of LP. ${ }^{5}$ Additionally, severe and possibly fatal disorders, such as infection transmission, nerve root damage, and hernia of the brain, may also be associated with LP procedures. ${ }^{6}$

Infants and children are often infected by viral or bacterial infections that may spread to the CNS, causing primary or secondary infections. Extracting CSF using LP is one of the diagnostic measures. ${ }^{7}$ Although LP is a safe operation in skilled hands, some parents deny their consent for it to be performed on their children. ${ }^{8}$ There is no explanation of the factors involved in their lack of consent, and any inconsistencies with the nonconsenting parents may provide information on how to deal with them. ${ }^{8}$ Although the diagnosis of meningitis can only be made with an accurate examination of CSF, an obstacle to doing CSF examinations in children is the refusal of parents. ${ }^{8,9}$ The prevalence and some underlying reasons for parents' reluctance to consent to LP were recorded in a few studies. Parents' refusal to consider
LP will put the child in a difficult situation if a sudden febrile convulsion is suspected to be caused by meningitis. ${ }^{9}$ A limited number of studies have focused on evaluating the rate of parental rejection regarding LP. These studies showed that the rate of rejections was low in the United States, with only $5 \%,{ }^{10}$ and in Denmark, with only 7\%." The rate of LP rejections was high in the United Arab Emirates, with $44 \%,{ }^{8}$ and in East Asia, with $25-28 \% .^{9}$ Thus, depending on cultural values, location, and level of awareness, parental actions regarding the LP procedure may be different. ${ }^{12}$

However, concerns about performing LP examinations on adults are not well studied and need to be evaluated. In Saudi Arabia, a recent study by Abdulrahman et al.. aimed to assess the awareness and attitudes of people in the area of Riyadh, Saudi Arabia regarding LP. ${ }^{12}$ A significant combination between acceptable level of information and an acceptable level of attitude were found via a multivariate logistic regression. ${ }^{12}$ There was also a significant correlation according to age, notably 46 years and over. $^{12}$ The attitudes toward LP were markedly negative among those who feared injections in the lumbar region. There is a lack of sufficient public knowledge of the LP technique, and the general public knowledge suggests that LP is unacceptable. Increased health education could increase understanding of the clinical importance and value of LP and the potential complications. ${ }^{12}$ However, these studies failed to determine the participants' educational backgrounds and sources of information regarding LP. Knowledge of the significance of LP might affect the acceptance rate of LP among the community. In particular, there is no clear understanding among Saudi Arabian population of the LP procedure. Thus, the factors behind the low acceptance rate of LP need to be evaluated to help deal with LP refusal and to increase the benefits of using LP for diagnosis and treatment. The current study seeks to evaluate the comprehension of the medical laboratory students about general facets of LP, the common purpose of performing LP, and the acceptance rate of LP. It will also compare the status of the population that medically needs LP.The research will provide the common possible factors related to the acceptance rate of LP. 


\section{Methods}

\section{I Study Context}

The research was carried out at Taif University's College of Applied Medical Sciences (February and April 2010) in Taif, Saudi Arabia. The data were obtained from those students enrolled in the Clinical Laboratory Sciences (CLS) program.

\subsection{Data Collection}

An online questionnaire was developed for this cross-sectional analysis after an exhaustive review of the literature. A highly useful data collection method was developed and further improved through consultations with other experts in the field.

The questionnaire was then tested on a group of 25 participants, similar to the main study participants. Finally, improvements were made in order to strengthen and verify the questionnaire on the basis of the experts' evaluation in the pilot test. The questionnaire was developed in English. It was distributed to 200 students enrolled in the CLS program, and 176 responses were received, indicating an $88 \%$ response rate.

\subsection{Study Variables and Analysis}

The questionnaire consisted of 2 I questions, composed of different patterns, II of them answered by yes or no, with the remaining questions requiring different answers. The first section of questionnaire inquired about independent variables of participants' personal information such as age, gender, and health education level. The other sections of the questionnaire inquired about dependent variables included knowledge of the LP operation (e.g., purpose, process, time of operation, fear of LP operation), acceptance of LP, the possible risks associated with the operation, their information sources and the precautions surrounding LP. Participants were also questioned about their acceptance of LP and whether they would prefer to undergo prolonged diagnosis and treatments rather than undergoing LP. A univariate analysis was performed. The participants' answers were analyzed as follows: those who scored $65 \%$ or above had high knowledge, high acceptance, and high preference for other study variables; and those who scored below
$65 \%$ had low knowledge, low acceptance, and low preference for other study variables.

The Social Sciences Statistical System (SPSS version 21.0) was used as the method for data analysis. The findings were presented in tables and graphs. Data were analyzed in the form of percentages. The $t$-test was used to determine the significance of the difference in each study variable. A $P<0.05$ was considered statistically significant and a $P>0.05$ was considered statistically non-significant.

\section{Results}

\section{I Characteristics of Study Participants}

A total of 176 responses from students of the CLS program at Taif University were analyzed. Saudi participants accounted for $100 \%$ of the respondents, with $90 \%$ being male and $10 \%$ being female (Table I). Approximately $87.5 \%$ of participants were ages 18-25, 3.4\% were ages 26-35, and 9.1\% were ages 36-49 (Table I). The bulk of the sample (95.8\%) was undergraduate students and $4.5 \%$ had a higher education.

\subsection{Participants' General Knowledge of LP}

The participants were asked about their knowledge of LP, and about $81.8 \%$ of participants were aware of LP. This result was significantly higher compared to only $18.2 \%$ of participants who did not know about

Table I: Sociodemographic data of participants involved in the study

\begin{tabular}{llcc}
\hline \multirow{2}{*}{ Item } & Response & $\begin{array}{c}\text { Number } \\
(\mathbf{N})\end{array}$ & $\begin{array}{c}\text { Percentage } \\
\text { (\%) }\end{array}$ \\
\hline \multirow{3}{*}{ Nationality } & Saudi & 176 & $100 \%$ \\
\cline { 2 - 4 } & Non-Saudi & 0 & $0 \%$ \\
\hline Sex & Male & 4 & $10 \%$ \\
\cline { 2 - 4 } & Female & 172 & $80 \%$ \\
\hline Age & $18-25$ & 154 & $87.5 \%$ \\
\cline { 2 - 4 } & $26-35$ & 6 & $3.4 \%$ \\
\cline { 2 - 4 } & $36-45$ & 16 & $9.1 \%$ \\
\hline Social status & Single & 24 & $13.6 \%$ \\
\cline { 2 - 4 } & Married & 168 & $95.8 \%$ \\
\cline { 2 - 4 } Educational & Bachelors & 8 & $4.5 \%$ \\
\cline { 2 - 4 } level & Higher education & & $86.4 \%$ \\
\hline
\end{tabular}


LP $(P<0.05)$. The results showed that there is no significant difference among participants regarding their awareness of the purpose and the process of LP $(P>0.05)$. Almost $73.9 \%$ showed they had knowledge about the duration of time required to perform LP (Figure IA). The source of their information was also analyzed, $53.6 \%$ of participants got the information from their school or university; $19.7 \%$ from hospitals; II.3\% from media; and $15.4 \%$ from their families and friends (Figure IB). The second section of the study was focused on understanding the personal characteristics of the participants with regard to their fear of LP. Their knowledge about side effects and precautions surrounding the LP operation was also examined. The results showed significantly more awareness of side effects (87.5\%), compared to $12.5 \%$ of participants who were not aware of the side effects of LP $(P<0.05)$. In addition, $72.7 \%$ were also aware about the precautions of LP (Figure IC). About $73.9 \%$ of respondents expressed worry about experiencing LP, compared to $26.10 \%$ were willing to get LP $(P<0.05)$ (Figure IC).

The knowledge of the purpose of LP showed that 5 I. $28 \%$ of participants believed the purpose was for disease diagnosis followed by $25.64 \%$ for anesthesia, $19.66 \%$ for therapeutic purposes, and $3.42 \%$ for other reasons (Figure ID). The participants' experiences with LP as patients were also analyzed and the results showed that $72.9 \%$ of participants had never undergone LP, $16.7 \%$ had undergone it once, and II.I\% had undergone it twice (Figure IE).

\subsection{Participants'Acceptance of LP}

Analyzing the participants' acceptance of LP was an important parameter in understanding their

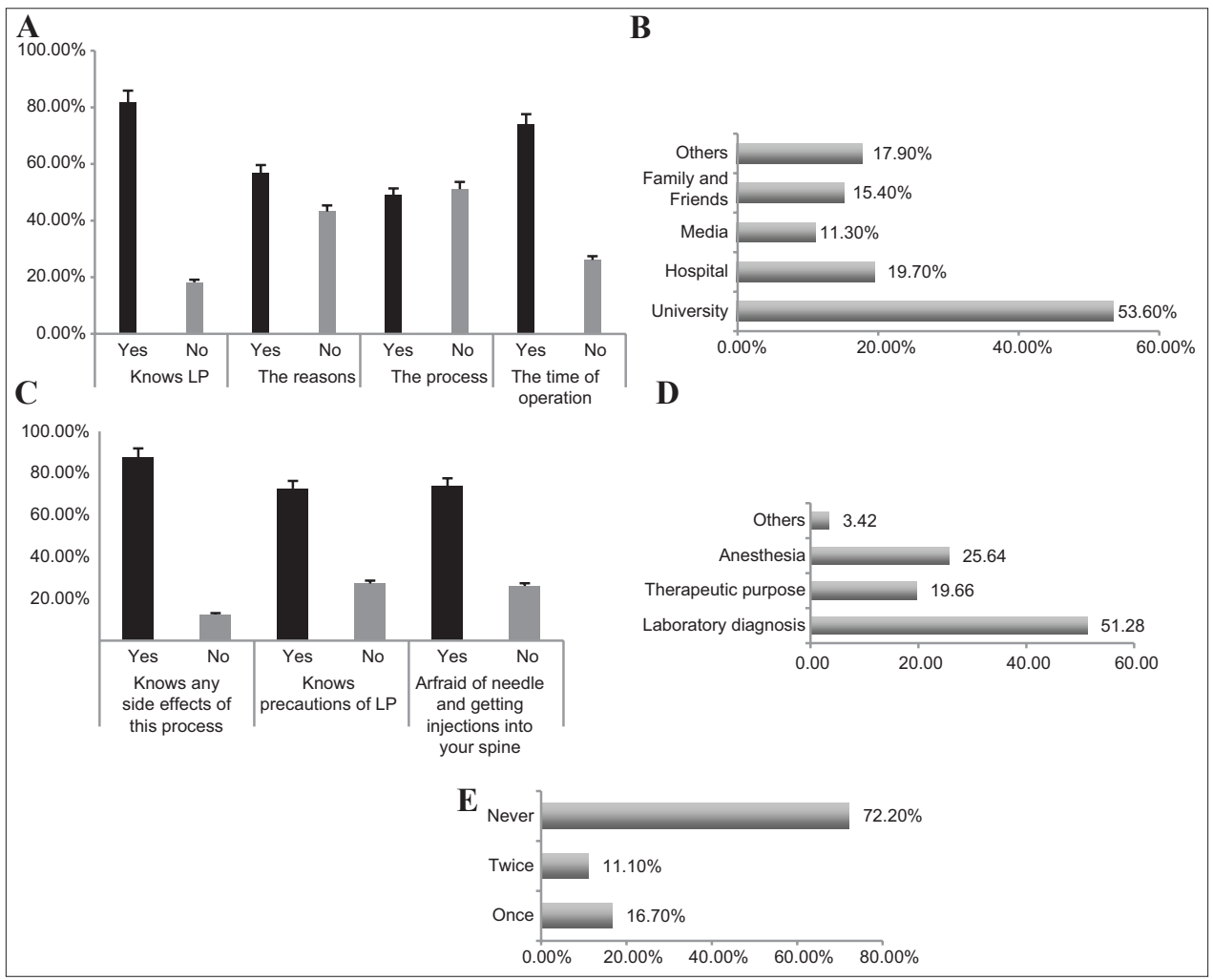

Figure I: Assessing the knowledge of LP among medically-educated individuals. A.The knowledge of the purpose, process, and duration of the LP operation. B. Participants' source of information related to LP, with $53.4 \%$ of participants learned their information from university. C. The rate of awareness regarding side effects and precautions surrounding LP was relatively significant. In addition, $73.9 \%$ of participants were afraid of needle injection. D. Responses regarding the purpose of LP. E. Responses regarding whether participants had undergone LP before 
awareness regarding LP.The participants were initially asked about their general acceptance of LP.The results revealed that $65.9 \%$ showed acceptance while $34.1 \%$ did not $(P<0.05)$. In addition, the results showed that there was no significant difference within the population with regard to their knowledge of the safety of LP and whether the operation should be avoided $(P>0.05)$. To better analyze their acceptance of LP, the participants were subsequently asked about their response to a hypothetical approval of LP if it was recommended by their doctor. About $72.7 \%$ of participants would prefer to not do LP even if it was recommended by their doctor. This result was significantly larger than $27.3 \%$ who would do LP based on their doctor's request $(P<0.05)$.Additionally, there was no significant differences among participants with regards to undergoing prolonged treatment rather than getting a diagnosis by LP $(P>0.05)$ (Figure 2A).

\subsection{Analysis of the Possible Side Effects of LP by Participants}

In assessing the participant's knowledge of the possible side-effects of LP, $70.5 \%$ thought that paralysis was a common side effect. The other participants believed that headaches (12.5\%), infections (8\%), no side effects (6.8\%), and other side effects (2.3\%) were associated with LP (Figure 2B).

\subsection{Analysis of the Precautions Surrounding LP by Participants}

The knowledge of the precautions surrounding LP was also analyzed. The results showed $84.10 \%$ of

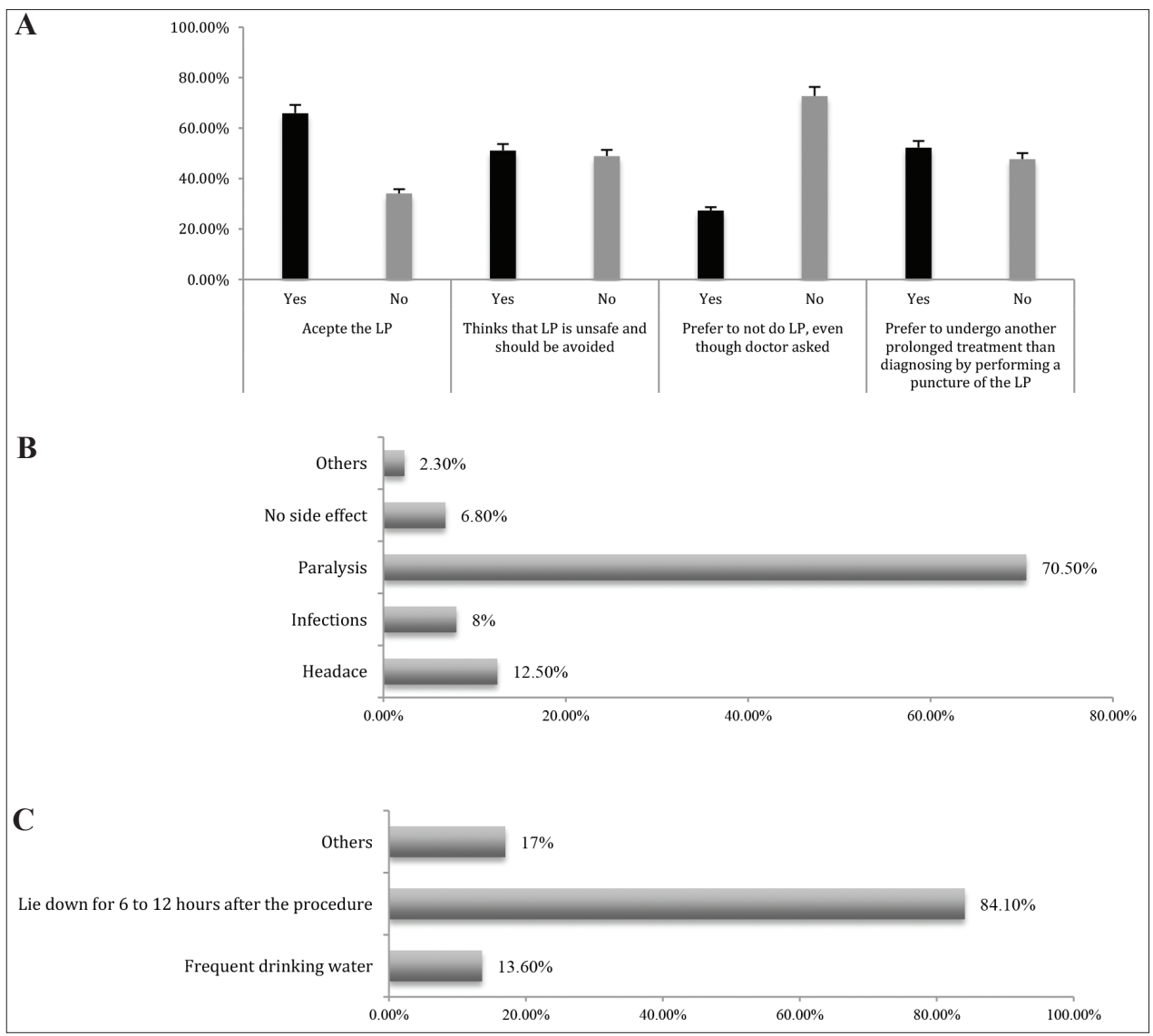

Figure 2: Evaluation of the acceptance rate of LP among medically-educated individuals. A.The general acceptance rate of LP was $65.9 \%$. When placed in the hypothetical scenario of a doctor's recommendation for LP, $72.7 \%$ preferred to not do LP. B. Responses regarding the complications of LP. The largest complication was paralysis (84.2\%). C. Responses regarding the precautions following LP 
participants believed that lying down for 6-12 hours after the procedure was a proper precaution, 13.6\% believed frequently drinking water was a proper precaution and $17 \%$ believed "Other" precautions were proper (Figure 2C).

\section{Discussion}

Diagnosis of critical diseases needs extensive processes to aid in accurate diagnosis and treatment. Patients need more information about LP as a diagnostic/therapeutic process. In particular, meningitis cannot be diagnosed without an appropriate analysis of CSF. Parental rejection of LP due to fear is a common issue that makes medical diagnosis and careful treatment more complicated. ${ }^{9}$ While this problem is common in Saudi Arabia, there are few studies that deal with acceptance of LP, and most of these studies were focused on parental concerns. Therefore, the current study seeks to evaluate the rate of understanding and acceptance of LP among clinical laboratory students at Taif University, Saudi Arabia.

The result of the current study showed that $81.8 \%$ of participants were aware of LP. This result was substantially higher than just $18.2 \%$ who did not know about LP.This result is the line with the study by Sulaiman et al.. who showed approximately $82 \%$ of the staff of a public university in Malaysia were aware of LP. ${ }^{13}$ They showed that the level of education and the profession of the participants were closely correlated with both the information and the attitude toward LP. Academic workers and Master's or PhD students were likely to be aware of LP. ${ }^{13}$ The study is not about the general public perception but instead the specific understanding of clinical laboratory students. ${ }^{12}$ In contrast, the study of Borhani-Haghighi et al.. showed that low awareness of LP of $92.6 \%$ was highly prevalent and the patients included in their study also had a negative attitude towards LP (63\%). ${ }^{14}$ The results of current study showed a good relationship between the level of education and profession and the knowledge of LP. The participants showed that their knowledge on the purpose, the process, and the time duration of LP was relatively moderate to high. To correlate our participants' informational background, an overview was also done of the origins of their information, showing that $53.6 \%$ of participants obtained information from their schools or colleges; $10.7 \%$ from hospitals; $13.25 \%$ from the media; and $13.7 \%$ from their family and friends. The significance of having the information come from universities found in the current study would improve the understanding of LP advantages and may reduce the refusal of LP.

In the current study, the participants provided data on their understanding of the common purposes of LP. Their understanding of the reasons for LP were: disease diagnosis (68.2\%); anesthesia (34.1\%); therapeutic purposes (26.1\%); and other reasons (4.5\%). This result indicated the high knowledge of the purpose of LP among our study participants. This correlated with the fact that primary care physicians also conduct LP for the following purposes: CNS diseases, anesthetics, oncology, and geriatric drugs. The results of participant's knowledge about side effects of and precautions required after LP showed there was high awareness of side effects (87.5); $72.7 \%$ were also aware about the required precautions for LP.

The participants' acceptance rate of LP was studied at two levels to identify the factors affecting their acceptance. The first level related to their general acceptance in the event that they are in good health and do not need this procedure The second level aimed to measure the rate of acceptance of those who were in the event that they were in a situation that required LP as recommended by their doctors. The results from the first level showed that almost $65.9 \%$ of respondents showed their general acceptance of LP compared to $34.1 \%$ of participants who did not accept LP. In contrast, when the participants were hypothetically recommended an LP by a doctor, $72.7 \%$ preferred to not do LP.This result was significantly greater than $27.3 \%$ who would do LP based on their doctor's recommendation. However, there were no significant differences among participants with regard to preferring prolonged treatment over diagnosis by LP. Several possible factors were analyzed regarding the rate of LP acceptance. It is well known that fear and anxiety will lead to medical treatment being avoided, leading to adverse health effects. This fear is known as 
needle phobia at excessive and serious levels. This is part of a psychiatric DSM-5 disorder called "phobia by blood injector". ${ }^{15}$

Analysis of the side effects that participants thought were related with LP was essential to assessing their refusal rate. The results showed that $70.5 \%$ of participants believed that paralysis is a common side effect.The other participants thought that headaches ( $12.5 \%)$, infections (8\%), no side effects $(6.8 \%)$, and other side effects $(2.3 \%)$ were to be expected. These results were different from published data regarding the complications of LP. It has been shown that headache, pneumonia, weakness of the lower limbs, sudden hematoma, bleeding and leakage of the brain from the site of puncture, nerve damage, or brain hernias are possible LP complications. ${ }^{7}$ Moreover, the LP procedures are rarely associated with serious or fatal disorders, such as infection transmission, nervous radical damage, and brain hernia. ${ }^{8}$ Knowledge of precautions following LP was also analyzed. Reasonable precautions were believed to be: lying down for 6-12 hours after the operation (84.1\%), drinking water frequently (13.6\%), and other precautions (I7\%).

\section{Conclusion and Implications for Translation}

Overall, the study showed a high rate of LP knowledge among medical laboratory students regarding purpose, process, and duration of LP operation. However, the refusal of LP in our study was linked to factors related to the misconception that the LP procedure is significant. In most participants, fear of injection and side effects, such as paralysis, were key reasons for the refusal of LP. This research can be of practical significance in other contexts that discuss this problem. Although this study was based on medical-student participants, the results indicate a need for improved LP awareness, such as academic seminars and training sessions, for the general public. Consequently, relevant LP-related lessons in national health programs need to be considered.The Ministry of Health could, via social media and health campaigns, raise awareness about the importance of the LP process dispel myths about complications linked to LP.

\section{Compliance with Ethical Standards}

Conflicts of Interest: The authors declared no potential conflicts of interest with respect to the research, authorship, and/or publication of this article. Financial

Disclosure: The authors received no financial support for the research, authorship, and/or publication of this article. Ethics Approval: The Ethics Board of College of Applied Medical Sciences of Taif University in Saudi Arabia approved the study prior to data collection. The online questionnaire explaining the purpose of the study and clarifying that the data was only used for academic research and was treated confidentially. Only the participants who agree to be involved in study were filled out the questionnaire. Acknowledgements: The authors would like to thank all the respondents for the time and patience they have spent completing the questionnaire.

\section{Key Messages}

There is a high understanding of the purpose, process and duration of the LP procedure among medical students.

- Fears of injection and side effects, including paralysis, are the main reasons for LP rejection.

- There is a need to have comprehensive knowledge of LP, such as whom academic seminars and training workshops.

\section{References}

I. Hrishi AP, Sethuraman M. Cerebrospinal fluid (CSF) analysis and interpretation in neurocritical care for acute neurological conditions. Indian J Crit Care Med. June 20 I9;23(suppl 2):SI I5-SII9.

2. Roos KL. Lumbar puncture. Semin Neurol. 2003;23(I):105-II4.

3. Seehusen DA, Reeves MM, Fomin DA. Cerebrospinal fluid analysis. Am Fam Physician. 2003;68(6): I1031108.

4. Engelborghs S, Niemantsverdriet E, Struyfs H, et al. Consensus guidelines for lumbar puncture in patients with neurological diseases. Alzheimers Dement (Amst). 2017;8:1 II-1 26.

5. Williams J, Lye DC, Umapathi T. Diagnostic lumbar puncture: minimizing complications. Intern Med J. 2008;38(7):587-59।. 
6. Rastogi S, Bhandari R, SharmaV,PandeyT.Neurological complications following spinal anaesthesia in a patient with congenital absence of lumbar vertebra. Indian J Anaesth. 20।4;58(4):484-486.

7. Young $\mathrm{N}$, Thomas M. Meningitis in adults: diagnosis and management. Intern Med J. 20I8;48(II):12941307.

8. Narchi H, Ghatasheh G, Hassani NA, Reyami LA, Khan $\mathrm{Q}$. Comparison of underlying factors behind parental refusal or consent for lumbar puncture. World J Pediatr. 20 I3;9(4):336-34I.

9. Ling SG, Boey CC. Lumbar puncture refusal in febrile convulsion. Singapore Med J. 2000;4 I ( I0):485-488.

10. Jacobson DM, Marx JJ, Dlesk A. Frequency and clinical significance of Lyme seropositivity in patients with isolated optic neuritis. Neurology. |99|;4|(5):706-7| I.

II. Frederiksen JL, Larsson HB, Olesen J. Correlation

Author Query???

AQI: Kindly provide the Funding Support and Disclaimer text of magnetic resonance imaging and CSF findings in patients with acute monosymptomatic optic neuritis. Acta Neurol Scand. 1992;86(3):317-322.

12. Aldayel AY, Alharbi MM, Almasri MS, Alkhonezan SM. Public knowledge and attitude toward lumbar puncture among adults in Riyadh, Saudi Arabia: a cross-sectional study. SAGE Open Med. 2019;7:2050312119871066.

13. Wan Sulaiman W, Muhamad Saliluddin S, Ong Y, et al. A cross sectional study assessing the knowledge and attitudes toward lumbar puncture among the staff of a public university in Malaysia. Clin Epidemiol Glob Health. 2018; 6(I): 29-33.

14. Borhani-Haghighi A, Rezaei R, Etemadi S, Ghaem H, ShariatA. Knowledge and attitudes of Iranian patients with regard to lumbar puncture. Neurosciences. 2009; |4(4):360-363.

15. Battle DE. Diagnostic and Statistical Manual of Mental Disorders (DSM). Codas. 2013;25(2):191-192. 